\title{
Actualités des anticorps monoclonaux dans les maladies monogéniques aujourd'hui
}

Adeline Bauvois ${ }^{1}$, Mélusine Larivière ${ }^{3}$, Hervé Watier ${ }^{2,3}$, François Maillot ${ }^{1,3}$ remontent aux années 2000 et de nombreux essais sont désormais en cours. Les anticorps monoclonaux anti-(interleukine) IL-1 $\beta$ ont profondément transformé la prise en charge des maladies auto-inflammatoires en modulant la composante inflammatoire et en diminuant le risque d'amylose secondaire; les anticorps monoclonaux anti-TNF- $\alpha$ et anti-IL-6 sont également prescrits dans ces maladies. Dans le syndrome hémolytique et urémique atypique lié à des défauts de régulation de la voie alterne du complément, l'éculizumab, un anticorps monoclonal anti-C5, a permis d'améliorer le pronostic rénal des patients traités. Plus récemment, le lanadélumab, un anticorps monoclonal anti-kallicréine plasmatique, est venu renforcer l'arsenal thérapeutique des angiœdèmes héréditaires et le burosumab, un anticorps monoclonal anti-FGF23, celui du rachitisme hypophosphatémique lié à I'X. Ces exemples illustrent bien l'importance de I'utilisation des anticorps monoclonaux dans la prise en charge des maladies monogéniques, l'intérêt de considérer cette option thérapeutique dans ce domaine et la nécessité de poursuivre des recherches. <

Les maladies monogéniques sont des maladies génétiques liées à des mutations d'un seul gène, de transmission autosomale dominante ou récessive, liée à I'X, ou mitochondriale. Elles sont nombreuses (plus de 6000 ), mais rares et de gravité variable. Les anticorps monoclonaux thérapeutiques (AcM) sont utilisés depuis les années 2000 dans un nombre croissant de maladies monogéniques. Leurs mécanismes d'action sont nombreux, par neutralisation de médiateurs, antagonisme

de récepteurs, remplacement enzymatique, ou activité agoniste, ce qui permet leur utilisation dans des maladies aussi variées et différentes que l'hémophilie, les angiœdèmes héréditaires, les maladies auto-inflammatoires et bien d'autres. Certains AcM ont obtenu une autorisation de mise sur le marché (AMM), à l'instar de l'émicizumab (anti-facteurs $I X a / X$ ) approuvé en 2018 dans l'hémophilie $A$ [1], et I'alirocumab et l'évolucumab (anti-PCSK9 [proprotein convertase. subtilisin kexin type 9]) dans les hypercholestérolémies familiales [3, 4]. Ces anticorps sont évoqués dans d'autres articles de ce numéro de médecine/sciences. Dans cette revue, nous avons choisi de développer le cas du burosumab, indiqué dans le rachitisme hypophosphatémique lié à I'X [2], ainsi que décrire trois autres situations pour lesquelles I'utilisation des AcM représente une avancée thérapeutique importante : les maladies auto-inflammatoires, les syndromes hémolytiques et urémiques (SHU) atypiques et les angiœdèmes héréditaires.

Sont regroupées sous le terme de maladies auto-inflammatoires, des maladies systémiques ayant une forte composante clinique et biologique inflammatoire, caractérisées par des anomalies génétiques de l'immunité innée, sans élévation des titres d'auto-anticorps ou présence de lymphocytes $T$ spécifiques d'antigènes $[5,6]$. Les maladies les mieux connues sont celles qui font intervenir l'interleukine-1 $\beta$ (IL$1 \beta$ ), telles que la fièvre méditerranéenne familiale (FMF) caractérisée dès 1997, les cryopyrinopathies (CAPS) avec mutations sur le gène NLRP3 (NOD-like receptor family, pyrin domain-containing 3), le déficit en mévalonate kinase et le TRAPS (TNF receptor-associated periodic fever syndrome). L'utilisation des AcM a pour but de contrôler les 
symptômes liés à l'inflammation, mais également de prévenir la complication grave qu'est l'amylose AA (amyloid-associated) secondaire à I'inflammation chronique. Les AcM anti-IL-1 $\beta$ sont utilisés en première intention puis, en cas d'échec, il est possible de recourir aux AcM anti-TNF- $\alpha$ ou aux inhibiteurs de I'IL-6 ou de son récepteur (anti-IL-6 et anti-IL-6R $\alpha$ ). Le canakinumab (anti-IL-1 $\beta$, Ig[immunoglobuline] Gl humanisée) possède une AMM depuis 2009 dans les CAPS. II a obtenu en 2017 une extension d'AMM dans les formes sévères de TRAPS ou de déficit en mévalonate kinase, et dans la FMF résistante à la colchicine [7]. L'adalimumab (IgGl), l'étanercept (fusion de l'ectodomaine du TNFR2 [tumor necrosis factor receptor 2] avec la région Fc d'lgGl) et l'infliximab (un AcM chimérique ayant une région Fc d'IgGl humaine) peuvent également être utilisés dans le TRAPS et la FMF [8]. Dans la FMF, en cas de résistance à la colchicine, les AcM anti-TNF- $\alpha$ ont principalement montré un intérêt en cas d'atteinte articulaire ou de maladie inflammatoire chronique de l'intestin [9, 10].

Les AcM sont également utilisés dans les microangiopathies thrombotiques (MAT) dues à des anomalies génétiques du complément, également appelées SHU atypiques. Les MAT sont définies par une anémie hémolytique mécanique avec présence de schizocytes ${ }^{1}$, une thrombopénie et des défaillances d'organes qui peuvent mettre en jeu le pronostic vital. Ce sont des maladies rares déclenchées par une activation non contrôlée de la voie alterne du complément qui entraîne un état proinflammatoire et procoagulant, avec la formation de microthrombi plaquettaires dans les vaisseaux de petits calibre et l'apparition de lésions endothéliales dans la microcirculation rénale [11]. L'atteinte rénale est prédominante avec une évolution vers l'insuffisance rénale terminale dans la majorité des cas dans les deux ans [12]. La C3 convertase de la voie alterne ( $C 3 b B b)$ génère à nouveau du C3b qui peut s'associer à une nouvelle molécule de facteur $B$ et créer une boucle d'amplification, ou s'associer à la C3 convertase ellemême pour former une $\mathrm{C} 5$ convertase ( $\mathrm{C} 3 \mathrm{bBbC} 3 \mathrm{~b}$ ) capable de protéolyser le $\mathrm{C} 5$ et d'initier la formation du complexe d'attaque membranaire. Comme l'activation de la voie alterne est initiée en continu par hydrolyse spontanée du C3, un strict contrôle de la C3 convertase alterne est indispensable. Le principal facteur qui en a la charge est le facteur $\mathrm{H}$ : il agit en dissociant le facteur B et en favorisant l'action du facteur I qui inactive le C3b. Des régulateurs cellulaires tels que le MCP (membrane cofactor protein, CD46), participent également à l'inactivation de $\mathrm{C} 3 \mathrm{~b}$. Des mutations perte de fonction du gène codant le facteur $\mathrm{H}$ ont été décrites dès 1998 [13]. D'autres mutations à l'origine de SHU atypiques ont ensuite été identifiées, notamment des mutations perte de fonction dans les gènes codant le facteur I et ceux codant CD46 ainsi que des mutations gain de fonction dans les gènes codant le $\mathrm{C3}$ ou le facteur B. Actuellement, la mutation causale est retrouvée chez près de $60 \%$ des patients [14] et les anomalies les plus fréquentes concernent le facteur $\mathrm{H}$. La prise en charge des patients atteints de SHU atypique reposait initialement sur les échanges plasmatiques et les transfusions de plasma frais congelé, dont l'effet n'était que suspensif et limité à l'atteinte rénale. La mise à disposition de l'écu-

Les schizocytes sont des hématies fragmentées présentes dans la circulation sanguine. lizumab et l'obtention d'une AMM en septembre 2012 dans l'indication de SHU atypique en première intention [15], a totalement bouleversé la prise en charge et le devenir des malades. Cet anticorps monoclonal neutralise le C5 en l'empêchant d'être clivé par les C5 convertases [16], ce qui le rend incapable d'initier la formation du complexe d'attaque membranaire. Les patients sous traitement présentent cependant une sensibilité accrue aux infections à méningocoques. La vaccination tétravalente antiméningococcique (sérogroupes $A, C$, $Y$ et W135) associée au vaccin contre le sérotype $B$ est donc obligatoire et une antibioprophylaxie peut être nécessaire. Les obstacles restent aujourd'hui le coût très élevé du traitement, qu'on pourrait cependant réduire en adaptant la posologie aux concentrations circulantes [17] ou en recourant à un biosimilaire [18], les difficultés pour définir une durée optimale de traitement et les effets secondaires infectieux [19]. Un nouvel anticorps anti-C5, le ravulizumab (ALXN1210), dont la demi-vie sérique est plus longue que l'éculizumab et qui permet des injections plus espacées, ne devrait pas tarder à bénéficier d'une AMM. Des anticorps dirigés contre d'autres cibles sont également en phase avancée de développement clinique, tels que I'OMS721, un anti-MASP2 (mannose-binding lectinassociated serine protease 2) [20].

Un troisième exemple est celui du lanadélumab pour le traitement prophylactique des angiœdèmes héréditaires de type I (déficit quantitatif en inhibiteur du Cl, Cl-INH) ou de type II (déficit qualitatif en Cl-INH). Les angiœdèmes héréditaires sont liés à des mutations du gène SERPINGI (serpin family G member 1), qui code le Cl-INH, un inhibiteur de protéase, notamment chargé d'inhiber la kallicréïne plasmatique et d'empêcher son action sur le kininogène de haute masse moléculaire et la libération de bradykinine qui en résulte. La libération excessive de bradykinine provoquée par ce déficit entraîne une augmentation de la perméabilité vasculaire qui provoque des œdèmes sous-cutanés ou des muqueuses pouvant menacer le pronostic vital. Des traitements des crises et prophylactiques existent mais avec une tolérance variable comme pour le danazol. Le lanadélumab est un anticorps monoclonal qui neutralise la kallicréine; il a été testé contre placebo en essai de phase III et permet de réduire le nombre de poussées [21]. II a reçu l'ATU (autorisation transitoire d'utilisation) en administration sous-cutanée en France en septembre 2018 pour la prévention des crises récurrentes d'angiœdème héréditaire de type I et II chez les patients âgés de plus de 12 ans, lorsque les traitements indiqués dans la prévention de routine sont inefficaces ou indisponibles [22]. 
Enfin, l'exemple du burosumab illustre la possibilité d'utiliser les AcM dans des pathologies monogéniques non immunologiques. En effet, cet AcM est indiqué depuis 2018 dans le traitement des enfants et adolescents présentant un rachitisme hypophosphatémique lié à I'X, non répondeurs ou intolérants aux traitements conventionnels. L'hypophosphatémie liée à I'X (XLH) est causée par une mutation du gène PHEX (phosphate-regulating endopeptidase homolog $X$-linked), codant une endopeptidase qui régule l'expression du fibroblast growth factor 23 (FGF23) [23, 24], notamment dans l'os. L'excès de FGF23 exerce au niveau du rein des effets indépendants sur l'expression du co-transporteur $\mathrm{Na}^{+} / \mathrm{PO}_{4}^{-}$, entraînant un défaut de réabsorption du phosphate dans les cellules tubulaires proximales rénales, ainsi qu'une diminution de la synthèse du $1,25(\mathrm{OH})_{2} \mathrm{D}$, métabolite actif de la vitamine $D$, par modulation de ses enzymes de synthèse et de dégradation [25]. Le traitement standard consiste en une supplémentation en sels de phosphore et en vitamine $D$, mais ce traitement est lourd (plusieurs prises par jour, à vie) et de nombreux patients présentent une forme résistante à la vitamine $D$. Le burosumab est une IgGl qui neutralise le FGF23, permettant la normalisation des taux de phosphate et de vitamine $D$ et une amélioration des symptômes. II présente notamment l'avantage d'une longue demi-vie, favorable à l'observance chez les jeunes patients.

Ces exemples illustrent bien l'importance de l'utilisation des anticorps monoclonaux dans la prise en charge des maladies monogéniques, l'intérêt de considérer cette option thérapeutique dans ce domaine et la nécessité de poursuivre des recherches pour en isoler de nouveaux. $\diamond$

\section{SUMMARY}

Monoclonal antibodies for monogenic diseases : a 2019 update

Monogenic diseases are rare genetic diseases but they are numerous and display a highly variable degree of severity. First uses of monoclonal antibodies to treat monogenic diseases started in the 2000's and many clinical trials are ongoing. Anti-IL-1 $\beta$ therapies have greatly modified the outcome of auto-inflammatory diseases by modulating inflammatory response and reducing the risk of secondary amyloidosis. AntiTNF- $\alpha$ are also used in such diseases. In atypical hemolytic and uremic syndrome due to deficiencies in the control of alternative complement pathway, eculizumab, an anti-C5 monoclonal antibody, has improved renal outcome in treated patients. More recently, lanadelumab, an anti-plasma kallikrein antibody, has reinforced the therapeutic arsenal in hereditary angioedema and burosumab, anti-FGF23, that of X-linked hypophosphatemia. Such examples reflect the importance of monoclonal antibody therapy of monogenic diseases, the interest of considering such an option as well as the need for future researches. $\diamond$

\section{LIENS D'INTÉRÊT}

Les auteurs déclarent n'avoir aucun lien d'intérêt concernant les données publiées dans cet article.

\section{RÉFÉRENCES}

1. Avis de la commission de transparence - Hemlibra (émicizumab). http://www.roche.fr/content/ dam/roche_france/fr_FR/doc/Produits/Hemlibra/Hemlibra_Col_11-07-2018.pdf. Accès au site $12 / 01 / 2019$
2. Cryvista (burosumab). Résumé des caractéristiques du produit. https:// www.ema.europa.eu/documents/product-information/crysvita-eparproduct-information_fr.pdf

3. Repatha. Arrêté du 8 février 2018 modifiant la liste des spécialités pharmaceutiques remboursables aux assurés sociaux. Legifrance. https:// www.legifrance.gouv.fr/eli/arrete/2018/2/8/SSAS1801874A/jo/texte/fr

4. Praluent. Arrêté du 19 janvier 2018 modifiant la liste des spécialités pharmaceutiques agréées à l'usage des collectivités et divers services publics. Legifrance. https://www.legifrance.gouv.fr/eli/arrete/2018/1/19/ SSAS1801114A/jo/texte/fr

5. Manthiram K, Zhou Q, Aksentijevich I, et al. The monogenic autoinflammatory diseases define new pathways in human innate immunity and inflammation. Nat Immunol $2017 ; 18: 832-42$.

6. Georgin-Lavialle S, Rodrigues F, Hentgen V, et al. Clinical overview of autoinflammatory diseases. Rev Med Interne 2018 ; 39 : 214-32.

7. Avis de la commission de transparence - ILARIS (canakinumab). https:// www.has-sante.fr/portail/upload/docs/evamed/CT-16220_ILARIS_PIC_EI_ Avis3_CT16220.pdf

8. Akgul 0 , Kilic $\varepsilon$, Kilic $G$, et al. Efficacy and safety of biologic treatments in Familial Mediterranean Fever. Am J Med Sci 2013 ; 346 : 137-41.

9. Georgin-Lavialle S, Hentgen V, Stankovic Stojanovic K, et al. Familial Mediterranean fever. Rev Med Interne 2018 ; $39: 240-55$.

10. Hentgen V, Grateau G, Kone-Paut I, et al. Evidence-based recommendations for the practical management of familial Mediterranean fever. Semin Arthritis Rheum $2013 ; 43: 387-91$.

11. Fakhouri F, Zuber J, Frémeaux-Bacchi V, et al. Haemolytic uraemic syndrome. Lancet 2017 ; $390: 681-96$.

12. Goodship THJ, Cook HT, Fakhouri F, et al. Atypical hemolytic uremic syndrome and C3 glomerulopathy: conclusions from a kidney disease: improving global outcomes (KDIGO) controversies conference. Kidney Int 2017; 91 : 539-51.

13. Warwicker P, Goodship TH, Donne RL, et al. Genetic studies into inherited and sporadic hemolytic uremic syndrome. Kidney Int $1998 ; 53: 836-44$.

14. Fremeaux-Bacchi V, Fakhouri F, Garnier A, et al. Genetics and outcome of atypical hemolytic uremic syndrome: a nationwide French series comparing children and adults. Clin J Am Soc Nephrol 2013 ; 8 : 554-62.

15. Avis de la commission de transparence - soliris (eculizumab). https:// www.has-sante.fr/portail/upload/docs/application/pdf/2012-10/ soliris_19092012_avis_ct12290.pdf

16. Brachet $G$, Bourquard T, Gallay N, et al. Eculizumab epitope on complement C5: Progress towards a better understanding of the mechanism of action. Mol Immunol 2016; 77 : 126-31.

17. Gatault P, Brachet G, Ternant D, et al. Therapeutic drug monitoring of eculizumab: Rationale for an individualized dosing schedule. mAbs $2015 ; 7$ : 1205-11.

18. A randomized, double-blind, single-dose, 3 -arm, parallel group study to determine the pharmacokinetic similarity of ABP 959 and Eculizumab (Soliris Registered Trademark) in healthy male subjects. ACTRN12616000509460 https://www.anzctr.org.au/Trial/Registration/TrialReview.aspx?id=369851

19. Ricklin D, Mastellos DC, Reis ES, et al. The renaissance of complement therapeutics. Nat Rev Nephrol 2018 ; 14 : 26-47.

20. Safety and efficacy study of OMS721 in patients with atypical hemolytic uremic syndrome. https://clinicaltrials.gov/ct2/show/NCT03205995.

21. Banerji A, Busse P, Shennak M, et al. Inhibiting plasma kallikrein for hereditary angioedema prophylaxis. N EnglJ Med 2017 ; 376 : 717-28.

22. Atu-Takhzyro (lanadelumab). https://ansm.sante.fr/var/ansm_site/ storage/original/application/aacfb94a0608503e6bb38250904ced7f.pdf

23. Francis $F$, Hennig $S$, Korn $B$, et al. A gene (PEX) with homologies to endopeptidases is mutated in patients with $X$-linked hypophosphatemic rickets. Nat Genet $1995 ; 11: 130$.

24. White KE, Evans WE, O'Riordan JLH, et al. Autosomal dominant hypophosphataemic rickets is associated with mutations in FGF23. Nat Genet $2000 ; 26: 345-8$.

25. Shimada T, Hasegawa H, Yamazaki Y, et al. FGF-23 is a potent regulator of vitamin D metabolism and phosphate homeostasis. J Bone Miner Res 2004 ; $19: 429-35$.
TIRÉS À PART

F. Maillot 